\title{
A Restriction on the Topology of Cauchy Surfaces in General Relativity
}

\author{
C. W. Lee \\ Department of Mathematics, Lancaster University, Lancaster LA1 4YL, England
}

\begin{abstract}
It is shown that, in a globally hyperbolic and geodesically complete space-time, a part of a partial Cauchy surface that is bounded by a uniformly convex sphere is compact and simply connected.
\end{abstract}

\section{Introduction}

There are several indications that we might reasonably disregard the possibility that the universe has anything other ${ }^{1}$ than a very simple topological structure. Geroch [1] has shown that an asymptotically simple and empty space-time is homeomorphic to $\mathbb{R}^{4}$. A result by Hawking ${ }^{2}$ on future asymptotically predictable space-times would seem to limit the possible topology of those space-times. There have also been some results on the possibility of the topology of space changing with time $[2,3,4]$.

It would be difficult to prove anything about the topology of space near to singularities, due to the arbitrariness associated with them. As the universe is expected to be globally past incomplete it would also be difficult to say anything about its topology as a whole without some uniformity principle. For these reasons we limit ourselves to a part of space that can be "enclosed by" a wellbehaved sphere. There is no restriction on the size of the sphere. We show that geodesic incompleteness is associated with topological peculiarities in such a part of space. That such incompleteness should occur separate from either collapsed objects or the beginning or end of the universe is objectionable.

The theorems are only proved for space-times with global Cauchy surfaces. There seems to be little reason to suppose that firstly the universe has one ${ }^{3}$, and secondly the results cannot be proved without one. It is a serious disadvantage, however, as the results are intended to be applicable to non-global topology without reference to, possibly unrealistic, global properties.

E.g., multiple connectedness

Proposition 9.2.1 of Reference [5]

See, for example, Reference [5] 
We firstly give a definition of a uniformly convex sphere. We suppose such a sphere to be embedded in a spacelike hypersurface. We show, under certain conditions, that the sphere divides the surface into two parts, that is there is no connection between the part of the hypersurface outside the sphere and the part inside, except through the sphere itself. We also establish that the part of the surface enclosed by the sphere is compact and simply connected.

The proofs follow in parts the singularity theorems of Hawking and Penrose [5]. Definitions are in accordance with [5] unless otherwise stated. We shall use $\dot{I}^{+}(X)$ for the boundary of the future of $X$, but $\partial_{Y} X$ for other topological boundaries.

\section{Definitions}

A uniformly convex sphere in a space-time is the image of a spacelike $C^{2}$ embedding of $S^{2}$, such that one congruence of null vectors orthogonal to it is converging. This definition should be compared with that of a closed trapped surface [5]. The latter requires both sets of null vectors to be converging; if this is the case for a uniformly convex sphere we may apply the following to either set of vectors. For this paper we will assume that the converging null vectors are future directed, as the theorems are otherwise time symmetric. We will always use $C$ to denote a uniformly convex sphere.

Suppose $C$ is contained in a partial Cauchy surface $S$. It will divide a small neighbourhood of itself in that surface into two parts, though it may not so divide the whole of $S$. The part that lies on the same side of $C$ as the converging null vectors we will call, loosely, the inside neighbourhood. Thus "a curve in $S$ that ends on the inside of $C$ " is a curve that reaches $C$ by passing through its inside neighbourhood.

We can now define the part of $S$ enclosed by $C$ as:

$$
A(C, S)=C \cup\left\{\begin{array}{c}
x \in S: \\
\text { There exists a curve in } S \text { from } x \text { to the } \\
\text { inside of } C \text { that does not cross } C
\end{array}\right\} .
$$

This may of course be the whole of $S$.

The set of future inextensible null geodesics with past endpoints on $C$, and there tangent to the converging null vectors, we will call $K(C)$.

\section{Theorems}

All the theorems proved, except Theorem 6, require the existence of a Cauchy surface, $\mathscr{H}$, for the space-time $(M, g)$, which we will assume implicitly. Only sketch proofs are given here. Detailed proofs are available from the author on request.

$V$ will be a timelike vector field on $M[1]$, and $\phi$ the map that takes points of $M$ along the integral curves of $V$ to where they intersect $\mathscr{H}$. We will only need $\phi$ to be continuous, and to map open sets to open sets.

Theorem 1. Suppose $(M, g)$ is null geodesically complete, and the energy condition [5] holds for null vectors. Suppose that $C$ is contained in $\mathscr{H}$, and that $A(C, \mathscr{H}) \neq \mathscr{H}$. Then $A(C, \mathscr{H})$ is compact. 
Outline of Proof. Let $T=\dot{I}^{+}(\mathscr{H} \backslash A(C, \mathscr{H})) \backslash(\mathscr{H} \backslash A(C, \mathscr{H})) . T$ will be generated by segments of members of $K(C)$, as $C=\partial_{\mathscr{H}} A(C, \mathscr{H})$. That $T$ is compact can be shown by a proof closely analogous to one contained in Theorem 1, Chapter 8.2, of [5]. As $\phi$ is continuous, $\phi(T)$ is compact.

$T$ has no edges, except at $C$, so $\phi(T)$ can have no boundary in $\mathscr{H}$, except at $C$. Thus $\phi(T)=A(C, \mathscr{H})$ and $A(C, \mathscr{H})$ is compact.

The C Covering Manifold. We need to define a covering manifold of $M$ in which $C$ divides any partial Cauchy surface that contains it into two parts. This is done in the appendix. We describe here a more intuitive construction.

Take a countably infinite number of copies of $M$, and order them with the integers. Cut each one at $\phi^{-1} \phi(C)$. Rejoin them by attaching the outside of $\phi^{-1} \phi(C)$ on the $n^{\text {th }}$ copy to the inside of $\phi^{-1} \phi(C)$ on the $(n+1)^{\text {th }}$ copy. If $\phi^{-1} \phi(C)$ did not divide $M$ into two parts, we will have connected all the copies of $M$ together to give a covering manifold, $M_{C}$, of $M$.

The sphere which joins the $(-1)^{\text {th }}$ copy to the $0^{\text {th }}$ copy will be called $C_{C}$. The union of all the positive numbered copies of $M$ will be called $B_{C}$. $\mathscr{H}_{C}, V_{C}$, and $\phi_{C}$ will correspond to $\mathscr{H}, V$, and $\phi$.

Theorem 2. Suppose $(M, g)$ is null geodesically complete, and the energy condition holds for null vectors. Suppose that $C$ is contained in $\mathscr{H}$. Then $A(C, \mathscr{H}) \neq \mathscr{H}$.

Outline of Proof. Suppose $A(C, \mathscr{H})=\mathscr{H} . M_{C}, C_{C}, \mathscr{H}_{C}$ satisfy the conditions of Theorem 1 , so $A\left(C_{C}, \mathscr{H}_{C}\right)$ is compact. However, it consists of an infinite number of copies of $\mathscr{H}$, all those contained in positive numbered copies of $M$, and so cannot be compact.

We have now established that a uniformly convex sphere in a Cauchy surface encloses a compact portion of that surface. We can extend the result to general spacelike hypersurfaces $^{4}$ using the same covering manifold. For Theorems 3-6 it will be assumed that $(M, g)$ is timelike and null geodesically complete, the energy condition holds for non-spacelike vectors, and the generic condition [5] holds.

Theorem 3. Suppose that $S$ is an acausal set containing $C$, with edge $(S)=C$, and $S$ on the inside of $C$. Then $S$ is compact.

Outline of Proof. Let $T_{C}=\dot{I}^{+}\left(C_{C}, B_{C}\right)$. Because $\phi_{C}^{-1} \phi_{C}\left(C_{C}\right)$ separates $M_{C}$ into two parts, $T_{C}$ will be generated by segments of members of $K\left(C_{C}\right)$. It will thus be compact, as will $\phi_{C}\left(T_{C}\right)$ and $\mathscr{H}_{C} \cap B_{C}$.

Define $S_{C}$ as the component of the image of $S$ in $M_{C}$ that contains $C_{C}$. To show that $S_{C}$ is compact we must show that $\phi_{C}\left(S_{C}\right)=\mathscr{H}_{C} \cap B_{C}$. If this is not the case, the boundary of $\phi_{C}\left(S_{C}\right)$ in $\mathscr{H}_{C} \cap B_{C}$ is non-empty. Let $\gamma$ be an integral curve of $V_{C}$ that passes through it. To the past it enters $D^{-}\left(T \cup\left(\mathscr{H}_{C} \cap B_{C}\right)\right)$ and does not leave

$4 \quad$ See [5] for the relationship between these and partial Cauchy surfaces 
it. By an argument similar to one contained in a theorem of Hawking and Penrose ${ }^{5}$, there exists a future trapped set, and thus, by that theorem, we get a contradiction. Hence $\phi_{C}\left(S_{C}\right)=\mathscr{H}_{C} \cap B_{C}$, and $S_{C}$, and hence $S$, is compact.

Theorem 4. Suppose $C$ is contained in a partial Cauchy surface $S$. Then $A(C, S) \neq S$, and is compact.

Outline of Proof. $A(C, S) \neq S$ by a similar argument to Theorem 2. It is compact by Theorem 3 .

We now need to define a covering manifold, $M_{\mathscr{H}}$, of $M$ in which the part of a partial Cauchy surface enclosed by $C_{\mathscr{H}}$ is simply connected. This is done in the Appendix. It can be seen that $M_{\mathscr{H}}$ is the universal covering manifold of $M$.

Theorem 5. Suppose $C$ is contained in a partial Cauchy surface $S$. Then $A(C, S)$ is simply connected.

Outline of Proof. Let $S_{\mathscr{H}}$ be the component of the image of $S$ in $M_{\mathscr{H}}$, containing $C_{\mathscr{H}}$. If $A(C, S)$ were not simply connected, $A\left(C_{\mathscr{H}}, S_{\mathscr{H}}\right)$ would not be compact. However, $M_{\mathscr{H}}, C_{\mathscr{H}}$, and $S_{\mathscr{H}}$ satisfy the conditions of Theorem 4, so it is compact.

Theorems 3 and 5 show that $A(C, S)$ is simply connected and compact. We can construct a topological 3-manifold, $A^{+}$, by joining $A(C, S)$ and $B^{3}$, the 3-ball, along their boundaries. It will be compact, as $B^{3}$ and $A(C, S)$ are compact. It will be simply connected as $B^{3}$ and $A(C, S)$ are simply connected and the common boundary is path connected. $A^{+}$is a homotopy 3 -sphere, and if the Poincare conjecture $^{6}$ is true it is homeomorphic to $S^{3}$. In that case we can deduce that $A(C, S)$ is homeomorphic to $B^{3}$.

The following theorem, concerning the part of space not enclosed by $C$, can be proved without the existence of a Cauchy surface.

Theorem 6. Suppose the chronology condition holds on $M$, and $C$ is contained in a partial Cauchy surface $S$. Then $S \backslash A(C, S)$ is non-compact, or empty.

Outline of Proof. Suppose it is compact. Then $E^{+}(S \backslash A(C, S))$ is contained in $(S \backslash A(C, S)) \cup \bigcup_{k \in K(C)} k$, which is compact. Hence $S \backslash A(C, S)$ is a future trapped set. This leads to a contradiction ${ }^{6}$.

\section{Conclusions}

It has been shown that, in a geodesically complete space-time that has a global Cauchy surface, a part of a partial Cauchy surface that is bounded by a uniformly convex sphere is compact and simply connected. If the Poincare conjecture is true, it is homeomorphic to $B^{3}$.

Suppose space did have some topological peculiarity. If it were on a scale smaller than that of the whole universe, and the space-time around it were not too curved, it could be "enclosed by" a uniformly convex sphere ${ }^{7}$. From Theorem 1 ,

Theorem 2, Chapter 8.2, of Reference [5]

See, for example, Reference [6]

It is interesting to ask whether any homotopy class of spheres contains a uniformly convex one 
we might expect that at least one of the ingoing null geodesics orthogonal to it would not be extensible past its conjugate point. Thus we might expect singularities to appear in space within a time of the same order as the diameter of $C^{8}$.

I am very grateful to Dr. C.T.J. Dodson and Dr. L.W. Flinn for many useful discussions.

\section{Appendix}

The Construction of the C Covering Manifold. Suppose $(M, g)$ has a uniformly convex sphere $C$. Choose $a \in C$, and let:

$$
M^{\prime}=\{(y, \gamma): y \in M \text {, and } \gamma \text { is a curve in } M \text { from } y \text { to } a\} .
$$

Let $n(\gamma)$ be the number of times the curve $\phi \circ \gamma$ crosses from $\phi(C)$ to its outside, minus the number of crossings to $\phi(C)$ from its outside. Let:

$$
\left(y_{1}, \gamma_{1}\right) \sim_{C}\left(y_{2}, \gamma_{2}\right) \text { iff } y_{1}=y_{2} \text { and } n\left(\gamma_{1}\right)=n\left(\gamma_{2}\right) \text {; }
$$

$\sim_{C}$ is an equivalence relation on $M^{\prime}$. It can be used $[2,5,8]$ to define a covering manifold, $M_{C}$, of $M$, which is a space-time locally isometric to $M$. Let $p_{C}: M_{C} \rightarrow M$ be the covering map.

The set of points of $M_{C}$ that are equivalence classes of points $(y, \gamma)$ of $M^{\prime}$ such that:

(1) $n(\gamma) \geqq 0 \quad$ we will call $B_{C}$

(2) $y \in \mathscr{H} \quad$ we will call $\mathscr{H}_{C}$

(3) $y \in C, n(\gamma)=0 \quad$ we will call $C_{C}$.

Let $V_{C}$ be the vector field induced by $V$. $\mathscr{H}_{C}$ is a Cauchy surface for $M_{C}$, as if $\alpha$ is an inextensible timelike curve in $M_{C}, p_{C} \circ \alpha$ is inextensible and timelike in $M$, so meets $\mathscr{H}$, and thus $\alpha$ meets $\mathscr{H}_{C}$. Let $\phi_{C}: M_{C} \rightarrow \mathscr{H}_{C}$ be the map induced by $V_{C}$. Clearly $\phi_{C}\left(B_{C}\right) \subset B_{C}$, and by a straightforward application of the definition of the topology on $M_{C}$ we can show that $\partial B_{C}=\phi_{C}^{-1} \phi_{C}\left(C_{C}\right)$.

The Construction of the $\mathscr{H}$ Covering Manifold. Suppose $(M, g)$ has a uniformly convex sphere $C$. Choose $a \in C$ and define $M^{\prime}$ as before. Define the covering manifold $M_{\mathscr{H}}$ by the equivalence relation:

$$
\left(y_{1}, \gamma_{1}\right) \sim_{\mathscr{H}}\left(y_{2}, \gamma_{2}\right) \text { iff } y_{1}=y_{2} \text { and } \phi\left(\gamma_{1}\right) \text { is homotopic to } \phi\left(\gamma_{2}\right) \text { in } \mathscr{H} \text {. }
$$

The set of points of $M_{\mathscr{H}}$ that are equivalence classes of points $(y, \gamma)$ of $M^{\prime}$ such that:

(1) $n(\gamma) \geqq 0$

(2) $y \in \mathscr{H}$

we will call $B_{\mathscr{H}}$

(3) $y \in C$; and $\gamma(t)=y, \forall t$ we will call $C_{\mathscr{H}}$.

As $C$ is simply connected, $C_{\mathscr{H}}$ consists of just one copy of $C . \phi_{\mathscr{H}}^{-1} \phi_{\mathscr{H}}\left(C_{\mathscr{H}}\right)=\partial B_{\mathscr{H}}$, and $\phi_{\mathscr{H}}\left(B_{\mathscr{H}}\right) \subset B_{\mathscr{H}}$, as before. $\mathscr{H}_{\mathscr{H}}$ is a Cauchy surface for $M_{\mathscr{H}}$. It is homeomorphic to the universal covering manifold of $\mathscr{H}$, hence simply connected.

8 The Kruskal extension of the Schwarzschild solution [7] has uniformly convex spheres, on Cauchy surfaces, whose interiors are non-compact (for instance, the spheres $v=0, r=$ constant $>2 M$, on the Cauchy surfaces $v=0$ ). A singularity occurs at time $\pi M$ after $v=0$ 


\section{References}

1. Geroch, R.P.: Space-time structure from a global view point. In: General Relativity and Cosmology, Proceedings of International School in Physics "Enrico Fermi", Course XLV 11(ed. R.K.Sachs), pp. 71-103. New York: Academic Press 1971

2. Geroch, R.P.: J. Math. Phys. 8, 782-786 (1967)

3. Yodzis, P.: Commun. math. Phys. 26, 39-52 (1972)

4. Yodzis, P.: Gen. Rel. Grav. 4, 299-307 (1973)

5. Hawking, S.W., Ellis, G.F.R.: The large scale structure of space-time. Cambridge: University Press 1973

6. Bing, R. H.: Ann. Math. 68, 17-37 (1958)

7. Kruskal, M.D.: Phys. Rev. 119, 1743-1745 (1960)

8. Hocking, J.G., Young, G.S.: Topology: Reading, Mass.: Addison-Wesley Publishing Company, Inc. 1961

Communicated by J. Ehlers

Received December 12, 1975; in revised form May 5, 1976 